\title{
Picosecond X-Ray Laser Interferometry of Dense Plasmas
}

\author{
R. F. Smith, ${ }^{1}$ J. Dunn, ${ }^{1}$ J. Nilsen, ${ }^{1}$ V. N. Shlyaptsev ${ }^{2}$ S. Moon, ${ }^{1}$ J. Filevich,${ }^{3}$ J. J. Rocca, ${ }^{3}$ M. C. Marconi, ${ }^{3,4}$ \\ J. R. Hunter, ${ }^{1}$ and T. W. Barbee, Jr. ${ }^{1}$ \\ ${ }^{1}$ Lawrence Livermore National Laboratory, Livermore, California 94551 \\ ${ }^{2}$ Department of Applied Science, University of California Davis-Livermore, Livermore, California 94551 \\ ${ }^{3}$ Department of Electrical and Computer Engineering, Colorado State University, Fort Collins, Colorado 80523 \\ ${ }^{4}$ Department of Physics, University of Buenos Aires, Buenos Aires, Argentina
}

(Received 13 February 2002; published 22 July 2002)

\begin{abstract}
We present the first results from picosecond interferometry of dense laser-produced plasmas using a soft x-ray laser. The picosecond duration and short wavelength of the $14.7 \mathrm{~nm}$ Ni-like Pd laser mitigates effects associated with motion blurring and refraction through millimeter-scale plasmas. This enables direct measurement of the electron-density profile to within $10 \mu \mathrm{m}$ of the target surface. A series of highquality two-dimensional (2D) density measurements provide unambiguous characterization of the time evolution in a fast-evolving plasma suitable for validation of existing 1D and 2D hydrodynamic codes.
\end{abstract}

DOI: 10.1103/PhysRevLett.89.065004

PACS numbers: 52.70.La, 52.50.Jm

Interferometry is a powerful tool for accurately diagnosing the 2D evolution of dense laser-produced plasmas. Over the years, visible and UV interferometry techniques have been developed to study different laser-plasma characteristics including profile steepening [1], exploding foils [2], filamentation, and beam guiding effects at high intensity [3]. For fast-evolving plasmas it is desirable that the duration of the probe pulse be short to obtain an effective snapshot of the density profile while reducing the effects of plasma motion blurring at the ablation front. For example, Attwood et al. successfully employed a 15 ps duration, $266 \mathrm{~nm}$ wavelength probe to determine density profile steepening close to the target surface [1]. Also, short wavelength, $\lambda$, high brightness probe beams allow for characterization of large plasmas at high density with less deleterious effects from free-free absorption $\left\{\propto \lambda^{3}[1-\right.$ $\exp (-h c / \lambda k T)]\}$ and refraction $\left(\propto \lambda^{2}\right)$ [4]. Da Silva et al. first demonstrated the technique in the extreme ultraviolet with the NOVA generated a $15.5 \mathrm{~nm} \mathrm{Ne}$-like Y collisional $\mathrm{x}$-ray laser, using a Mach-Zehnder interferometer with thin multilayer beam splitters [4]. The high brightness of the 350 ps probe enabled electron densities of $\sim 10^{21} \mathrm{~cm}^{-3}$ to be diagnosed in a large millimeter-scale $\mathrm{CH}$ plasma. Recently, Rocca and co-workers established the $46.9 \mathrm{~nm}$ Ne-like Ar capillary discharge $\mathrm{x}$-ray laser as an effective tabletop interferometric tool using Lloyd's mirror and Mach-Zehnder diffraction grating instrumentation with a pulse of 1.2 ns duration $[5,6]$.

Extending the interferometric capability simultaneously to the short wavelength regime and short pulse regime with high brightness has been an important goal. Chirped pulse amplification has established compact, picosecond and femtosecond, high-power laser drivers capable of generating short wavelength, transient gain Ne-like and Ni-like $\mathrm{x}$-ray lasers [7]. These $\mathrm{x}$-ray lasers have the desired picosecond pulse duration [8] with more than $10 \mu \mathrm{J}$ output, in a well-collimated beam $(\sim \operatorname{mrad})$ at high repetition rates $(1$ shot per 4 min) [9] and are well suited as interferometric probes. Their temporal and spectral properties are important for extending interferometric techniques into laser-plasma regimes, where large, dense, fast-evolving, short-scale-length plasmas may be characterized. In this paper, we present the first results using recent picosecond $\mathrm{x}$-ray lasers for interferometry of laser-produced plasmas. High-quality 2D density profiles of a laser-heated Al plasma are measured using a diffraction grating interferometer at different times, relative to the peak of the plasma-forming pulse, to within $10 \mu \mathrm{m}$ of the target surface. The experimental results are compared with $1.5 \mathrm{D}$ and 2D hydrodynamic simulations.

The Ni-like Pd $14.7 \mathrm{~nm}$ X-ray laser probe beam and the plasma to be studied were generated using three laser beams at $1054 \mathrm{~nm}$ wavelength from the COMET facility at LLNL [9]. The single pass saturated $x$-ray laser output of a few tens of $\mu \mathrm{Js}$ was achieved with an optical pumping combination of a 600 -ps-long pulse $(2 \mathrm{~J}, 2 \times$ $\left.10^{11} \mathrm{~W} \mathrm{~cm}^{-2}\right)$ and a $6 \mathrm{ps}\left(5 \mathrm{~J}, 7 \times 10^{13} \mathrm{~W} \mathrm{~cm}^{-2}\right)$ main heating pulse. Traveling wave irradiation along the $1.6 \mathrm{~cm}$ line-focus geometry was employed on a $1.25 \mathrm{~cm}$ polished slab Pd target to produce amplification in the axial direction. As shown in Fig. 1, the x-ray laser output was imaged and partially collimated by a normal incidence multilayer spherical mirror (S1), with $f=11.75 \mathrm{~cm}$, and routed via a $45^{\circ}$ multilayer mirror (M1), to the input path of the diffraction grating interferometer (DGI). The measured reflectivity, for unpolarized $\mathrm{x}$ rays, of these two $\mathrm{Mo} / \mathrm{Si}$ multilayer optics was 0.62 (S1) and 0.30 (M1) at the laser wavelength with a bandpass of $0.62 \mathrm{~nm}$ and $1.44 \mathrm{~nm}$, respectively.

The $\mathrm{x}$-ray laser is incident at $4.5^{\circ}$ grazing incidence onto a Au-coated 900 lines/mm diffraction grating (G1), where the grating blaze angle is compensated to give equal reflectivity $(0.25)$ for the 0 th order and -1 st order arms. The two beams are reflected at $2.6^{\circ}$ grazing incidence from 


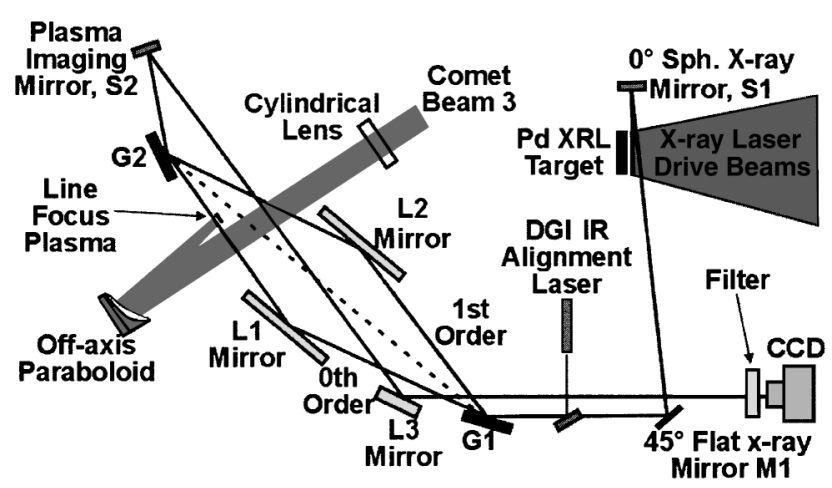

FIG. 1. Experimental setup for x-ray laser interferometry on the COMET laser.

30-mm-long Au-coated mirrors (L1, L2) and recombined at the second grating (G2) to form a single copropagating beam. The throughput of the instrument is estimated to be 0.0575 for each arm. A plasma heated by up to $2 \mathrm{~J}$ of energy in a $600 \mathrm{ps}, 1054 \mathrm{~nm}$ pulse, corresponding to a maximum intensity of $3 \times 10^{12} \mathrm{~W} \mathrm{~cm}^{-2}$, was produced in the 0 th order arm and was probed by the interferometer. A 0.6-cm-long line focus, where the width could be adjusted form 20 to $150 \mu \mathrm{m}$, was generated on a polished $\mathrm{Al} \mathrm{slab}$ target using a combination of a cylindrical lens, $f=$ $-200 \mathrm{~cm}$, and an off-axis paraboloid, $f=30 \mathrm{~cm}$. The relative delay between the arrival of the x-ray laser probe pulse to the peak of the plasma-forming beam was measured to within $100 \mathrm{ps}$ with a fast diode. The $\mathrm{x}$-ray laser could probe the plasma in the temporal range of $-1 \mathrm{~ns}$ to +2 ns relative to the peak of the 600 ps plasma-forming pulse by adjusting a delay arm in the plasma laser beam. The line-focus plasma was probed longitudinally by the $\mathrm{x}$-ray laser, thereby minimizing uncertainties in the interpretation of the interferograms arising from plasma gradients along the probe path. The plasma was imaged by a $25 \mathrm{~cm}$ focal length spherical multilayer mirror (S2) that was tilted $5^{\circ}$ from normal incidence. This beam was relayed to the CCD camera by reflection at grazing incidence from the third Au-coated mirror, L3. The interferogram images were recorded by a thinned back illuminated $1024 \times 1024 \mathrm{CCD}$ detector with $24 \times 24 \mu \mathrm{m}^{2}$ pixels. A $2000 \AA \mathrm{Zr} / 1000 \AA$ polyimide $\left(\mathrm{C}_{22} \mathrm{H}_{10} \mathrm{~N}_{2} \mathrm{O}_{5}\right)$ filter was placed in front of the CCD to block visible and UV light. The magnification of the imaging system was determined to be 9.94 by imaging a fine mesh at the target plane, giving a pixel-limited spatial resolution of $2.55 \mu \mathrm{m}$. The target angle was determined to be parallel to the 0th order $\mathrm{x}$-ray laser beam to better than $\pm 0.25^{\circ}$.

Interference fringes are generated when the two arms in the interferometer are of equal length and the temporal and spatial coherence conditions for the x-ray laser are met. The instrument was prealigned with an $827.1 \mathrm{~nm}$ diode laser selected to have a coherence length of $\sim 250 \mu \mathrm{m}$, similar to that of the $\mathrm{x}$-ray laser. By using the $\mathrm{x}$-ray laser beam with no plasma present, high-quality fringes, with visibility $V=\left(I_{\max }-I_{\min }\right) /\left(I_{\max }+I_{\min }\right)$, of $0.72 \pm 0.12$ were observed for a $700 \times 500 \mu \mathrm{m}^{2}(H \times V)$ region indicating excellent spatial coherence in the laser beam. A typical interferogram is shown in Fig. 2 for a 0.5 -cm-long Al target irradiated by a $0.6 \mathrm{~cm} \times 40 \mu \mathrm{m}$ line focus at an intensity of $3 \times 10^{11} \mathrm{~W} \mathrm{~cm}^{-2}$. The unperturbed peak-topeak fringe separation was set to $18 \mu \mathrm{m}$. The $\mathrm{x}$-ray laser probe time was $+0.7 \mathrm{~ns}$ after the peak of the $600 \mathrm{ps}$ pulse. The density sampled by the $\mathrm{x}$-ray laser occurs over a time interval dictated by the few picosecond duration of the $\mathrm{x}$-ray laser pulse and its transit time through the $0.5 \mathrm{~cm}$ plasma column ( $\sim 16.5 \mathrm{ps})$. The electron density, $n_{e}$ in $\mathrm{cm}^{-3}$, is related to the measured fringe shifts as $N_{\text {fringe }}=$ $6.68 \times 10^{-20} n_{e} L$, where $L$ is the length $(\mathrm{cm})$ of the plasma being probed by the $14.7 \mathrm{~nm} x$-ray laser [4]. Fringes are clearly visible less than $10 \mu \mathrm{m}$ from the target surface. The number of fringe shifts observed at this location corresponds to a peak density of $1.2 \times 10^{20} \mathrm{~cm}^{-3}$, with one fringe shift corresponding to an electron density, $n_{e}$, of $2.95 \times 10^{19} \mathrm{~cm}^{-3}$. The extent of the lateral expansion relative to the initial $40 \mu \mathrm{m}$ linewidth is seen to be significant. The bright area close to the target surface and peaked at $\sim 45 \mu \mathrm{m}$ is the first diffracted order from the 0th order arm, impinging on the imaging system.

Figure 3 shows on-axis density profiles at different probe times during and after the $600 \mathrm{ps}$ heater beam for a $0.1-\mathrm{cm}-$ long Al plasma irradiated by a $20 \mu \mathrm{m}$ linewidth. The target surface position is known to be $+8,-2 \mu \mathrm{m}$ for the $0.1 \mathrm{~cm}$ target lengths, primarily due to uncertainties in the target rotation. Fringe shifts are observed to less than $10 \mu \mathrm{m}$ from the target surface with measured electron densities

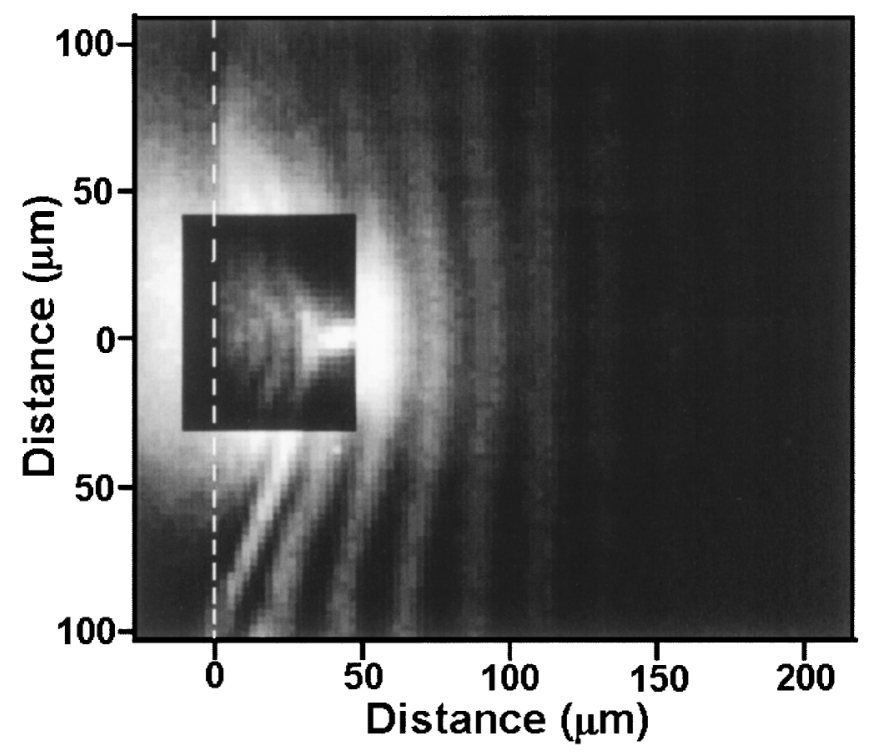

FIG. 2. Interferogram recorded at $\Delta t=0.7 \mathrm{~ns}$ from a 0.5 $\mathrm{cm}$-long Al plasma heated by a $0.4 \mathrm{~J}, 600 \mathrm{ps}$ pulse. The laser line focus is $0.6 \mathrm{~cm} \times 40 \mu \mathrm{m}$. The image was recorded on a 16-bit CCD camera. Two different gray scales are shown for clarity. 


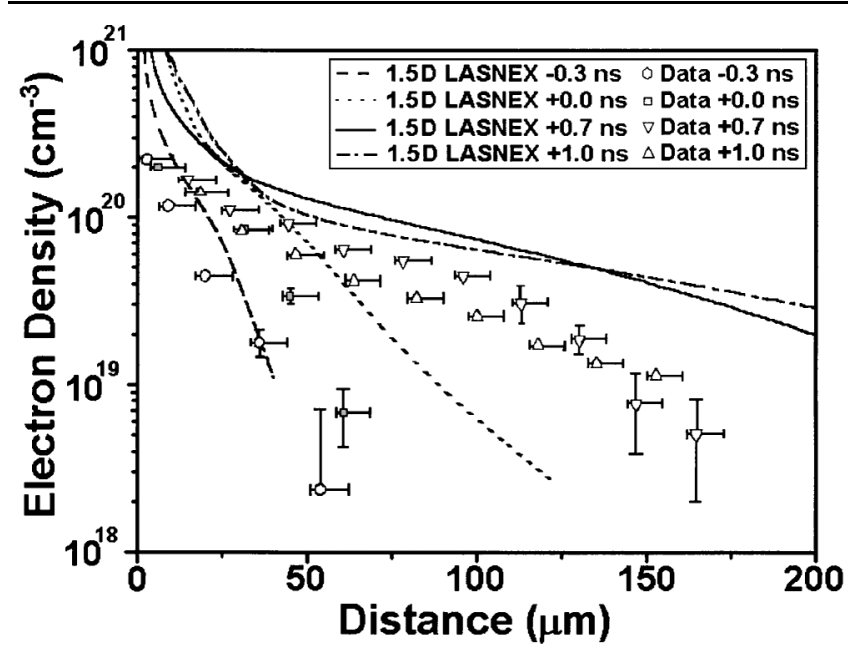

FIG. 3. Measured electron density profiled as a function of distance away from the target surface for different probe times compared with 1.5D LASNEX simulations.

up to $3 \times 10^{20} \mathrm{~cm}^{-3}$. This, the highest density experimentally reported in this paper, is not the limit of the $\mathrm{x}$-ray interferometric technique. The critical density for the $14.7 \mathrm{~nm}$ probe wavelength is $5 \times 10^{24} \mathrm{~cm}^{-3}$ and gives the ultimate limit to the density with which the x-ray laser can probe. The on-axis profiles in Fig. 3 show that the predicted $n_{e}$ increases rapidly within $5 \mu \mathrm{m}$ of the target surface. Experimental images indicate very fine fringe structure in this region, which are not well resolved by our present imaging setup. In addition, modeling predicts that, for a $2 \omega$ plasma-forming beam, higher electron densities in excess of $10^{21} \mathrm{~cm}^{-3}$ would be measured using the same experimental setup. The lowest measured electron density for this target length is limited to $2 \times 10^{18} \mathrm{~cm}^{-3}$ and is dependent on the minimum detection of the fringe shift. It can be seen at early times, $t=-0.3$ and $0 \mathrm{~ns}$, that the density scale length is short and the plasma has expanded to $60 \mu \mathrm{m}$ from the initial target surface. At later times, $t=+0.7$ and $1.0 \mathrm{~ns}$ when the plasma heating pulse is effectively off, the plasma scale length has relaxed, and the plasma has continued to expand to $160 \mu \mathrm{m}$. Shown alongside the data points are the predicted electron densities from LASNEX simulations [10]. The LASNEX simulations are one dimensional but include an expansion angle of $15^{\circ}$ in the direction perpendicular to the primary expansion (1.5D) so as to simulate the $2 \mathrm{D}$ effects associated with the narrow width of the line focus on the target. The code predictions give qualitatively good agreement in reproducing the plasma-density profile evolution. The overall trend, however, is for the simulations to show somewhat higher density at all times. The plasma planar expansion transitions to a cylindrical expansion at early times with our tight $20 \mu \mathrm{m}$ focal spot, which means that 2D effects are important at all times. Close to the target surface, the lateral expansion velocity of the plasma away from the initial laser focus was measured to be $7.5 \times$
$10^{6} \mathrm{~cm} / \mathrm{s}$ over the interval of probing. This is in agreement with the expected sound speed for a plasma electron temperature of $T_{e} \sim 80 \mathrm{eV}$. In addition, perturbations in the fringes away from the target surface indicated an expansion angle of $\pm 35^{\circ}$ at $t>0.5 \mathrm{~ns}$. This also confirms that $2 \mathrm{D}$ simulations are required to directly compare the various lateral expansion features observed in the experiment. Figure 4 shows a contour plot of a $2 \mathrm{D}$ LASNEX simulation and the corresponding experimental data at $\Delta t=0.5 \mathrm{~ns}$ with good quantitative agreement. Such modeling covers all phases of expansion leading to a plasma size much larger than the focal spot.

Refraction should be carefully considered in all interferometric analyses of plasmas and should not be neglected. Rays of the probe beam which initially propagate parallel to the target will be deflected and affect the interferograms in a number of ways. The refracted beams will have a slightly different path length, which results in a modified phase differential when these rays are combined with the 1 st order arm. Interpretation is further complicated by different electron densities sampled along the trajectory of the refracted rays. In addition, the angle of the phase front for the refracted rays will combine with the -1 st order arm leading to a contraction of the fringe separation [11].

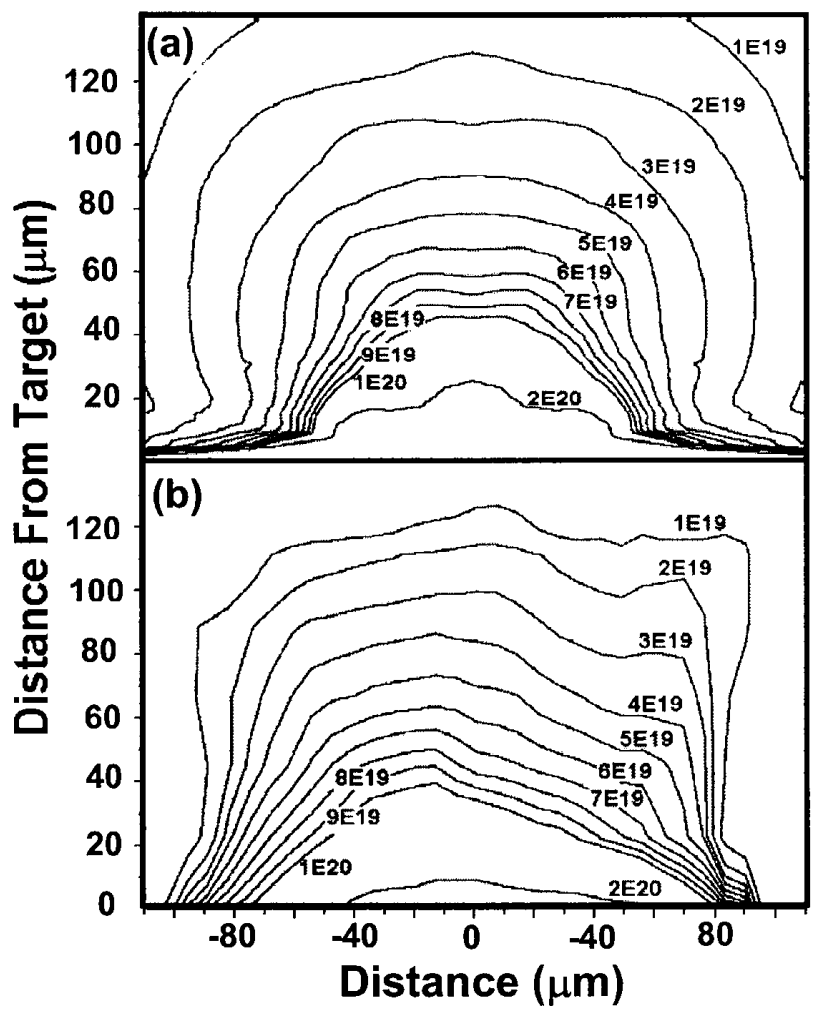

FIG. 4. (a) Simulated 2D electron-density profile using LASNEX. (b) Measured 2D electron-density profile $0.5 \mathrm{~ns}$ after the peak of the 600 ps duration plasma-forming beam. The error bars for distance away from the target are $-2 \mu \mathrm{m}$, $+8 \mu \mathrm{m}$. 




FIG. 5. 1D RADEX simulation of refraction at time of peak electron density versus $z$ for $0.1 \mathrm{~cm} \mathrm{Al}$ plasma. The calculated $n_{e}$ profile (solid line) is shown along with predicted observable electron density using the experimental setup, when refraction is considered over a $0.1 \mathrm{~cm}$ plasma (dashed line). The RADEX simulations modeled the experimental intensity described in Fig. 3 but implemented an $80 \mu \mathrm{m}$ linewidth to give better agreement to the $2 \mathrm{D}$ experimental plasma.

We systematically investigated the effect of refraction with experiments and simulations to verify our density measurements for $0.1 \mathrm{~cm}$ targets. The paraxial ray equation for the angle of refraction may be approximated as $\theta=$ $X n_{e}(z) / 2 n_{c} L$, where $X$ is the length of the target, $z$ is the distance away from the target, $n_{c}$ is the critical density, and $L$ is the density scale length given as $n_{e}(z)=$ $n_{e o} \exp (-z / L)$. We placed a $12.7 \mu \mathrm{m}$ spacing mesh in front of the plasma and backlit it with the 0th order arm. Laser shots for a $0.5 \mathrm{~cm} \mathrm{Al}$ target under the irradiation conditions of Fig. 2 show that the grid image, initially at 13.3 and $26 \mu \mathrm{m}$ from the target surface, was displayed a further 11 and $4.49 \mu \mathrm{m}$, respectively, by the plasma corresponding to deflection angles of 2.2 and $0.9 \mathrm{mrad}$, respectively. Therefore, under similar conditions for a $0.1 \mathrm{~cm} \mathrm{Al}$ target the $14.7 \mathrm{~nm}$ probe rays propagating $13.3 \mu \mathrm{m}$ from the target would undergo a $\sim 0.44 \mathrm{mrad}$ deflection. Overall, for the densities reported for $0.1 \mathrm{~cm}$ target lengths, refraction is expected to be negligible. Rays closer to the target and at higher density would have larger deflections, as shown in the 1D RADEX simulations [12] for the effect of refraction at $+0.5 \mathrm{~ns}$ (time of peak measured $n_{e}$ ) in Fig. 5. The calculated electron density and the expected density observable by interferometry when refraction effects are considered $(0.1 \mathrm{~cm}$ target length $)$ are plotted as a function of the initial distance away from the target surface. Refraction is taken into account by ray tracing through the millimeter long plasma while also considering the resulting distortion of the phase front. The effect of refraction contributes to a $<20 \%$ overesti- mation in electron density for $\sim 3 \times 10^{20} \mathrm{~cm}^{-3}$ at $z=$ $12 \mu \mathrm{m}$. The use of simulation and grid deflectometry measurements gives insight into the effects of refraction on the interferograms in a rigorous manner.

In conclusion, picosecond $\mathrm{x}$-ray laser interferometry has been successfully conducted for the first time. The combination of short wavelength, very large density limit, picosecond duration, and high source brightness shows that this is a powerful tool for diagnosing large, hot, dense plasmas. The robustness of the experimental design combined with the high repetition rate available, facilitates the capture of a series of 2D snapshots of the electron-density profile and the construction of an effective moving picture of the plasma evolution. Such information is valuable for the benchmarking of 2D hydrodynamic plasma physics codes. We have self-consistently determined the effect of refraction by simulations and experiment and its effect on the density measurements. Probing closer to the target surface will be possible by imaging with micrometer spatial resolution obtainable using a higher magnification and a higher resolution detector. This will be addressed in future experimental work to extend the technique to higher electron densities unattainable by other methods.

The support of Al Osterheld and Andy Hazi is greatly appreciated. The authors are pleased to acknowledge the technical contributions from Carl Bruns and Al Ellis. This work was performed under the auspices of the U.S. Department of Energy by the University of California Lawrence Livermore National Laboratory, through the Institute for Laser Science and Applications, under Contract No. W-7405-Eng-48 and by U.S. Department of Energy Grant No. DE-FG03-98DP00208. The development of the interferometer gratings was supported with a grant from the State of Colorado Photonics and Optoelectronics program.

[1] D. T. Attwood et al., Phys. Rev. Lett. 40, 184 (1978).

[2] M. D. Rosen et al., Phys. Rev. Lett. 54, 106 (1985).

[3] P. E. Young and P. R. Bolton, Phys. Rev. Lett. 77, 4556 (1996); P.E. Young et al., Phys. Rev. Lett. 81, 1425 (1998).

[4] L. B. Da Silva et al., Phys. Rev. Lett. 74, 3991 (1995).

[5] J. J. Rocca et al., Opt. Lett. 24, 420 (1999).

[6] J. Filevich et al., Opt. Lett. 25, 356 (2000).

[7] P. V. Nickles et al., Phys. Rev. Lett. 78, 2748 (1997); J. Dunn et al., Phys. Rev. Lett. 80, 2825 (1998).

[8] A. Klisnick et al., Phys. Rev. A 65, 033810 (2002).

[9] J. Dunn et al., Phys. Rev. Lett. 84, 4834 (2000).

[10] G. B. Zimmerman and W. L. Kruer, Comments Plasma Phys. Control. Fusion 2, 51 (1975).

[11] P. Celliers et al., Opt. Lett. 20, 1907 (1995).

[12] V. N. Shlyaptsev et al., Proc. SPIE Int. Soc. Opt. Eng. 2012, 111 (1993). 